\title{
The Effects of Genetic Relatedness on the Preterm Infant Gut Microbiota
}

\author{
Shen Jean Lim ${ }^{1,2}$, Miriam Aguilar-Lopez ${ }^{3} \mathbb{D}$, Christine Wetzel ${ }^{4}$, Samia V. O. Dutra ${ }^{1,5}$, Vanessa Bray ${ }^{6} \mathbb{D}$, \\ Maureen W. Groer 1,5 (D), Sharon M. Donovan ${ }^{3}$ (D) and Thao Ho ${ }^{6, *(D)}$ \\ 1 College of Nursing, University of South Florida, Tampa, FL 33612, USA; jx12581@miami.edu (S.J.L.); \\ sozoriod@utk.edu (S.V.O.D.); mgroer@usf.edu (M.W.G.) \\ 2 Rosenstiel School of Marine and Atmospheric Science, University of Miami, Miami, FL 33149, USA \\ 3 Division of Nutritional Sciences, University of Illinois Urbana-Champaign, Urbana, IL 61801, USA; \\ aguilarl@illinois.edu (M.A.-L.); sdonovan@illinois.edu (S.M.D.) \\ 4 Neonatology Division, Carle Hospital, Urbana, IL 61801, USA; Chris.Wetzel@carle.com \\ 5 College of Nursing, University of Tennessee, Knoxville, TN 37916, USA \\ 6 College of Medicine, University of South Florida, Tampa, FL 33612, USA; vtbray@usf.edu \\ * Correspondence: tho1@usf.edu; Tel.: +1-813-844-3437
}

Citation: Lim, S.J.; Aguilar-Lopez, M.; Wetzel, C.; Dutra, S.V.O.; Bray, V.; Groer, M.W.; Donovan, S.M.; Ho, T. The Effects of Genetic Relatedness on the Preterm Infant Gut Microbiota. Microorganisms 2021, 9, 278.

https://doi.org/10.3390/

microorganisms 9020278

Academic Editor: Claudio de Simone

Received: 22 December 2020

Accepted: 25 January 2021

Published: 29 January 2021

Publisher's Note: MDPI stays neutral with regard to jurisdictional claims in published maps and institutional affiliations.

\begin{abstract}
The preterm infant gut microbiota is influenced by environmental, endogenous, maternal, and genetic factors. Although siblings share similar gut microbial composition, it is not known how genetic relatedness affects alpha diversity and specific taxa abundances in preterm infants. We analyzed the $16 \mathrm{~S}$ rRNA gene content of stool samples, $\leq$ and $>3$ weeks postnatal age, and clinical data from preterm multiplets and singletons at two Neonatal Intensive Care Units (NICUs), Tampa General Hospital (TGH; FL, USA) and Carle Hospital (IL, USA). Weeks on bovine milk-based fortifier (BMF) and weight gain velocity were significant predictors of alpha diversity. Alpha diversity between siblings were significantly correlated, particularly at $\leq 3$ weeks postnatal age and in the TGH NICU, after controlling for clinical factors. Siblings shared higher gut microbial composition similarity compared to unrelated individuals. After residualizing against clinical covariates, 30 common operational taxonomic units were correlated between siblings across time points. These belonged to the bacterial classes Actinobacteria, Bacilli, Bacteroidia, Clostridia, Erysipelotrichia, and Negativicutes. Besides the influence of BMF and weight variables on the gut microbial diversity, our study identified gut microbial similarities between siblings that suggest genetic or shared maternal and environmental effects on the preterm infant gut microbiota.
\end{abstract}

Keywords: preterm infant; gut microbiota; human milk; twins; triplets

\section{Introduction}

A balanced infant gut microbiota is important for the maturation of intestinal functions [1], development of innate and adaptive immune responses [2], weight gain [3], growth [4], and even long-term health [5]. The preterm infant gut microbiota is influenced by gestational age, birth weight, and other clinical factors associated with preterm birth [6]. Compared to the term infant gut microbiota, the preterm infant gut microbiota is characterized by reduced microbial diversity, lower abundances of obligate anaerobes, dominance of potentially pathogenic Enterobacteriaceae (including Citrobacter, Escherichia, Enterobacter, Klebsiella, and Serratia, among others), and delayed colonization by commensal gut bacteria (such as Clostridium and Veillonella) typical of adult gut microbiota [4-11]. These deviations, collectively referred to as dysbiosis, are associated with increased susceptibility of infections and diseases, including necrotizing colitis (NEC) [11,12], bacteremia [13,14], late onset sepsis [15], impaired growth [4], and possibly other long-term health consequences [6].

The preterm infant gut microbiota is shaped by various therapeutic, environmental, and endogenous factors $[6,16]$. Therapeutic interventions include the administration of 
histamine-2-receptor blockers in infants [17], and, more significantly, the administration of prophylactic antibiotics in mothers $[18,19]$ and infants $[12,20]$. Maternal intrapartum antibiotic use is associated with increased Enterobacteriaceae abundances in the gut of preterm infants [19]. Similarly, postnatal antibiotic use in preterm infants increases Enterobacter abundances and decreases gut microbial diversity and Escherichia abundances [12,20]. Thus, antibiotic-mediated shifts in gut bacterial diversity likely contribute to gut dysbiosis in preterm infants and its associated adverse health effects, including NEC, sepsis, and even death [20]. Environmental influences on preterm infant gut microbiota include effects of delivery mode [11,19], enteral feeding of formula, breast milk, and/or human milk-based fortifier (HMF) [21-23], and relatively stronger effects of Neonatal Intensive Care Unit (NICU) exposure [7,24]. On the other hand, endogenous influences on preterm infant gut microbiota can include maternal physiology (such as stress, chronic disease, effects from smoking, and infections) [6,16,25], infant gut physiology, and immune system responses [26].

Host genetics, previously reported to influence adult [27-30] and healthy term infant microbiota [31], can also play a role in shaping preterm infant gut microbiota [12]. Previous studies reported similarities in gut microbiota composition between preterm twins and multiplets [12,32], although sample sizes were small ( $<15$ multiplet sets). Beyond gut compositional similarities, high-throughput sequencing has not been applied to identify specific effects of genetic relatedness on gut microbial diversity, including alpha diversity and bacterial abundances. Currently, it is also not known whether genetic similarities between preterm infant siblings are solely due to genetics or a combination of clinical factors, including antibiotics exposure, diet, maternal exposure, or NICU exposure. Largerscale, multi-center analyses comparing gut microbial abundances between multiplets and singletons within and between centers can advance our current understanding of genetic and environmental influences on the preterm infant gut microbiota. These findings have the potential to improve clinical care of preterm neonates.

In this study, we sought to examine preterm gut infant microbiota from multiplets and singletons admitted to two different neonatal intensive care units (NICUs) located in Tampa General Hospital (TGH; FL, USA) and Carle Hospital (Carle, IL, USA). We analyzed the clinical data and microbial diversity of stool samples collected before and after three weeks postnatal age to evaluate the effects of clinical factors and genetic relatedness on gut microbial alpha and beta diversity. We used the three-week postnatal age cutoff because the gut microbiota composition of predominantly preterm, low birthweight infants was previously shown to resemble the composition of age-matched, normal birthweight infants between 18 and 21 days of life [32].

\section{Materials and Methods}

\subsection{Enrollment and Sample Collection}

After approvals from the respective Institutional Review Boards, preterm infants were enrolled from two level III NICUs, TGH and Carle NICUs, between 2012-2017 and 2016 and 2018, respectively. The TGH NICU provides one room for each infant that is equipped with a refrigerator and a milk warmer. Twins and triplets in TGH were housed in two or three separate rooms, which often shared a removable wall. On the other hand, the 48-bed-capacity Carle Hospital NICU has an open bay unit configuration with multiple pinwheel layout. Each pinwheel contains three back-to-back bedspaces and a handwashing station. Several infants share a milk warmer and a refrigerator for prepared daily feedings. There are two private rooms reserved for rooming in prior to discharge. The Carle and TGH NICUs had similar antibiotic, feeding volume, and advancement practices. However, Carle provided an exclusive human milk diet for infants born $<28$ weeks with the use of HMF (Prolacta Bioscience, Industry, CA, USA) and TGH NICU only provided bovine-based fortifier (BMF; Similac ${ }^{\circledR}$ Human Milk Fortifier, Abbott Nutrition, Columbus, OH, USA) during the study period. 
The study inclusion criteria included birth weight $<1500 \mathrm{~g}$ or birth gestation $<33$ weeks. Infants with major chromosomal or congenital intestinal anomalies were excluded. Weekly infant stool samples were collected from diapers from enrollment until discharge. Samples were either placed on dry ice (Carle) or placed in a $-20{ }^{\circ} \mathrm{C}$ freezer (TGH), prior to storage at $-80{ }^{\circ} \mathrm{C}$ until DNA extraction and analysis. Demographic and perinatal data collected from the electronic medical records included delivery method, sex, postnatal age (days), corrected gestational age (weeks), days of antibiotic exposure, weeks on BMF/HMF milk fortifier, birth weight, current weight, weekly average weight gain velocity (g/day), and proportions of consumed mother's own milk, formula, and donor human milk (from the week prior to each stool sample collection).

\subsection{Sample DNA Extraction and Sequencing}

Total DNA from stool specimens in the Carle cohort was extracted using the QIAamp Fast DNA Stool Mini Kit (QIAGEN, Valencia, CA, USA) with the bead-beating method previously described [33]. The V3-V4 region of the 16S rRNA gene was amplified with dual-indexed primers (Supplementary Materials Table S1) and sequenced at the Roy J. Carver Biotechnology Center at the University of Illinois on the Illumina MiSeq $2 \times 250 \mathrm{bp}$ platform (San Diego, CA, USA). DNA extraction and library preparation protocols for the TGH stool samples were as previously published [22]. Briefly, total DNA was extracted using the QIAamp ${ }^{\circledR}$ PowerFecal DNA Kit (QIAGEN, Valencia, CA, USA; previously known as MoBio PowerFecal DNA kit). The V4 region of the 16S rRNA gene was amplified using modified 515F and 806R primers [22] (Supplementary Materials Table S1) and sequenced on Illumina's MiSeq $2 \times 300$ bp platform.

\subsection{Bioinformatics and Statistics}

Demultiplexed reads were quality filtered at a $Q=25$ cutoff using the Trim Galore! v0.4.4, (https: / / github.com/FelixKrueger/TrimGalore) wrapper package, then imported into QIIME2-2019.17 [34] and denoised with DADA2 [35] into amplicon sequence variants (ASVs; Supplementary Materials Table S2) without further trimming. Representative ASV sequences from the V3-V4 region (Carle cohort) and the V4 region (TGH cohort) of the 16S rRNA gene were deduplicated using CD-HIT v4.8.1 [36] at a global sequence identity threshold of $100 \%$, retaining only the longest sequence for each ASV. Because different primer sets were used for sequencing and the ASV sequences were mapped to the same SILVA v132 [37] reference database, ASV sequences were further clustered de novo into operational taxonomic units (OTUs) at 99\% identity, as recommended by Knight et al. [38]. The OTU table was filtered to remove OTUs with $<10$ total frequency and OTUs present in $<2$ samples. For phylogenetic diversity analysis, a tree containing all OTU sequences was generated using the SATé-Enabled Phylogenetic Placement (SEPP) fragment insertion method [39]. Taxonomies were assigned to OTU sequences using a naïve Bayes classifier trained on full-length 16S rRNA gene sequences from the SILVA v132 [37] database. Any OTUs with ambiguous taxonomic assignments were manually re-classified to the genus level using web blastn searches against NCBI's nt database [40], if the best hits unanimously matched to a single genus with $\geq 97 \%$ identity [41]. To maintain sample size consistency in all downstream analyses, samples with missing metadata were removed from the OTU table, followed by non-singleton samples with no corresponding sibling data at each time point.

Alpha diversity metrics were calculated from the rarefied OTU table (rarefied to 1232 OTUs per sample) using QIIME2 [34]. Among the alpha diversity metrics computed from the rarefied OTU count data $(n=1232)$, Shannon's diversity [42] was highly correlated with Pielou's evenness (Spearman rho $=0.85, p<0.001$ ) [43], while the number of observed OTUs was significantly correlated with Faith's phylogenetic diversity [44] (Spearman's rho $=0.80, p<0.001$ ). For downstream analyses, we chose Shannon's diversity [42] and Faith's phylogenetic diversity [44] as representative alpha diversity metrics. This is because null values of Pielou's evenness can arise from zero division [43] and 
because Pielou's evenness is derived from Shannon's diversity [42]. Compared to observed OTUs, Faith's phylogenetic diversity carries more information because it uses phylogenetic information to estimate richness [44]. Prior to downstream analyses, alpha diversity variables and clinical variables were transformed to a near-normal distribution using R's box cox function in the car package [45] or log-transformation (for the "days on antibiotic" variable with right-skewed distribution and zero values). Correlograms between variables before and after transformation were calculated, based on Spearman's correlations, and visualized using R's PerformanceAnalytics package (https:/ / github.com/braverock/PerformanceAnalytics; accessed on 10 August 2020).

To examine associations between alpha diversity and clinical variables, linear mixed effect models were fitted with R's lme4 [46] and nlme (https://CRAN.R-project.org/ package=nlme) packages using the maximized log-likelihood method. Clinical variables were input as fixed effect predictors and alpha diversity as the response variable. Model fit was evaluated using the likelihood ratio test, Aikake Information Criterion [47], and Bayesian Information Criterion. Statistically significant predictors $(p<0.05)$ were identified using ANOVA tests. The random structure of the models was tested using various combinations of family, individual, and zygosity, keeping the fixed effect predictors and response variable constant. Based on goodness-of-fit comparisons, family was chosen as the random effect in all models. Taxonomic abundance analyses and principal components analysis (PCA) were performed using the R package ampvis2 v2.6.5 on the rarefied count table, as recommended [48]. Prior to PCA, OTUs present in $<0.1 \%$ relative abundance in any sample were filtered, and the count data were transformed using the Hellinger method [49]. Shared OTU analyses were performed on unrarefied OTU counts using Venny v2.1 (https:/ / bioinfogp.cnb.csic.es/tools/venny/). Differential abundance analysis between TGH and Carle NICU samples was performed on unrarefied counts using the default parameters of Linear Discriminant Analysis (LDA) Effect Size (LEfSe) [50]. Beta diversity was calculated from unrarefied centered-log-ratio-transformed OTU counts using the Aitchison distance, which accounts for the compositionality of OTU count data [51]. Pairwise distances between siblings and non-siblings were compared using Mann-Whitney$\mathrm{U}$ tests and false-discovery-rate-adjusted $p$-values [52]. Spearman correlations of OTU abundances between siblings were calculated in R, using unrarefied OTU abundances that were residualized against clinical variables (covariates) with the umx_residualize function in the R package umx v1.9.1 (https:/ / www.rdocumentation.org/packages/umx).

\section{Results}

\subsection{Clinical Characteristics of Samples}

Eleven twin sets, 2 triplet sets, and 32 singletons were recruited from the Carle NICU, while 22 twin sets, 4 triplet sets, and 24 singletons were recruited from the TGH NICU (Supplementary Materials Table S2). From these individuals, a total of 264 stool samples were collected before and after three weeks postnatal age for this study (Table 1). Birth variables, including gestational age at delivery and weight were not significantly different between the TGH and Carle cohorts. Overall, the TGH cohort had a significantly higher proportion of male infants, and a significantly higher rate of cesarean delivery (Table 1). In the TGH cohort, the multiplets had greater birth gestational age and weight than those from Carle (Table 1). Two stool specimens, $\leq$ and $>3$ weeks postnatal age, were collected from each infant. Seven singletons from the TGH NICU were missing samples from $>3$ weeks postnatal age, while three twin sets from the TGH were missing samples from $\leq 3$ weeks postnatal age. Corrected gestational ages (sum of chronological and birth gestational ages) from both NICUs were not significantly different at the time of stool collection (Table 2). One infant from the TGH cohort was diagnosed with NEC after the collection of the included stool samples. An amount of $20 \%$ of the infants in the TGH cohort were diagnosed with culture-positive sepsis. None of infants from Carle cohort developed NEC or sepsis. Exposures to antibiotics and formula were greater in the TGH cohort compared to the Carle cohort (Table 2). 
Table 1. Demographics and clinical characteristics of the population at birth.

\begin{tabular}{|c|c|c|c|c|c|c|c|}
\hline \multicolumn{4}{|c|}{ Carle Hospital $n=118$ (44.7) } & \multicolumn{4}{|c|}{ Tampa General Hospital $n=146$ (55.3) } \\
\hline Cohort Type, $n,(\%)$ & $\begin{array}{c}\text { Multiplet } \\
n=54 \text { (45.8) }\end{array}$ & $\begin{array}{c}\text { Singleton } \\
n=64(54.2)\end{array}$ & $p^{1}$ & $\begin{array}{c}\text { Multiplet } \\
n=105 \text { (71.9) }\end{array}$ & $\begin{array}{c}\text { Singleton } \\
n=41(28.1)\end{array}$ & $p^{2}$ & $p^{3}$ \\
\hline $\begin{array}{l}\text { Gestational age at delivery, } \\
\text { weeks }\end{array}$ & $28.8(2.3)$ & $28.9(2.2)$ & 0.89 & $29.4(2.2)$ & $28.5(2.7)$ & 0.001 & 0.12 \\
\hline Birth weight, grams & $1246.9(443.1)$ & 1238.7 (399.2) & 0.93 & $1349.4(390.5)$ & $1118.7(199.3)$ & 0.0004 & 0.24 \\
\hline $\begin{array}{l}\text { Sex } \\
\text { Female, } n,(\%) \\
\text { Male, } n,(\%)\end{array}$ & $\begin{array}{l}30(55.6) \\
24(44.4)\end{array}$ & $\begin{array}{l}42(65.6) \\
22(34.4)\end{array}$ & 0.35 & $\begin{array}{l}42(40.0) \\
63(60.0)\end{array}$ & $\begin{array}{l}15(36.6) \\
26(63.4)\end{array}$ & 0.84 & 0.0006 \\
\hline $\begin{array}{l}\text { Type of delivery } \\
\text { Vaginal delivery, } n,(\%) \\
\text { Cesarean delivery, } n,(\%)\end{array}$ & $\begin{array}{c}4(7.4) \\
50(92.6)\end{array}$ & $\begin{array}{l}10(15.6) \\
54(84.4)\end{array}$ & 0.27 & $\begin{array}{c}4(3.8) \\
101(96.2)\end{array}$ & $41(100.0)$ & 0.48 & 0.007 \\
\hline $\begin{array}{l}\text { Number of placentas } \\
\text { Monochorionic twins, } n,(\%) \\
\text { Dichorionic twins, } n,(\%)\end{array}$ & $\begin{array}{l}12(22.2) \\
42(77.8)\end{array}$ & - & - & $\begin{array}{l}24(22.9) \\
81(77.1)\end{array}$ & - & - & 0.9 \\
\hline
\end{tabular}

Data expressed as means (SD) unless otherwise noted. $p^{1}$ : Comparisons between multiplet and singleton groups of Carle Hospital. $p^{2}$ : Comparisons between multiplet and singleton groups of Tampa General Hospital. $p^{3}$ : Comparisons between Carle Hospital and Tampa General Hospital. All statistical comparisons were based on the Kruskal-Wallis test for numerical data and the chi-square test for categorical data. Bold values indicate $p<0.05$.

Table 2. Demographics and clinical characteristics of the population at the time of stool collection.

\begin{tabular}{|c|c|c|c|c|c|c|c|c|c|}
\hline \multicolumn{5}{|c|}{ Carle Hospital $n=118(44.7 \%)$} & \multicolumn{5}{|c|}{ Tampa General Hospital $n=146(55.3 \%)$} \\
\hline \multirow[t]{2}{*}{ Cohort Type, $n,(\%)$} & \multicolumn{2}{|c|}{ Multiplet $n=54(45.8 \%)$} & \multicolumn{2}{|c|}{ Singleton $n=64(54.2 \%)$} & \multicolumn{2}{|c|}{ Multiplet $n=105(71.9 \%)$} & \multicolumn{2}{|c|}{ Singleton $n=41(28.1 \%)$} & \multirow[t]{2}{*}{$p^{*}$} \\
\hline & Early & Late & Early & Late & Early & Late & Early & Late & \\
\hline Corrected gestational age, weeks & $30.8(2.0)$ & $33.2(2.2)$ & $30.6(2.1)$ & $33.1(2.1)$ & $31.1(2.1)$ & $33.9(1.9)$ & $30.4(2.4)$ & $33.0(2.5)$ & 0.31 \\
\hline Postnatal age, days & $13.7(8.2)$ & $29.9(9.0)$ & $11.8(6.6)$ & $29.0(8.0)$ & $10.1(4.9)$ & $29.2(7.3)$ & $13.0(6.4)$ & $31.4(5.0)$ & 0.62 \\
\hline Current weight, grams & $\begin{array}{l}1388.8 \\
(441.4)\end{array}$ & $\begin{array}{l}1853.1 \\
(597.9)\end{array}$ & $\begin{array}{l}1369.3 \\
(438.2)\end{array}$ & $\begin{array}{l}1866.1 \\
(574.2)\end{array}$ & $\begin{array}{l}1358.2 \\
(376.7)\end{array}$ & $\begin{array}{l}1877.1 \\
(463.5)\end{array}$ & $\begin{array}{l}1194.0 \\
(283.5)\end{array}$ & $\begin{array}{l}1655.7 \\
(260.7)\end{array}$ & 0.5 \\
\hline Weight gain velocity, $\mathrm{g} / \mathrm{d}$ & $9.3(9.7)$ & $20.9(9.1)$ & $6.9(14.0)$ & $21.2(10.0)$ & $-1.4(12.3)$ & $17.8(6.7)$ & $1.1(15.5)$ & $17.1(4.0)$ & 0.001 \\
\hline Days on antibiotics, days & $0.5(1.5)$ & $0.2(1.0)$ & $0.7(1.5)$ & $0.0(0.2)$ & $2.7(2.7)$ & $3.3(3.7)$ & $4.2(3.6)$ & $4.5(3.7)$ & $<0.0001$ \\
\hline Bovine milk fortifier, weeks & $1.4(1.0)$ & $3.3(1.8)$ & $1.1(1.0)$ & $2.8(2.1)$ & $0.9(0.8)$ & $3.7(1.1)$ & $1.5(1.0)$ & $3.9(0.8)$ & 0.16 \\
\hline Proportion of mother's own milk & $0.83(0.28)$ & $0.77(0.32)$ & $0.63(0.37)$ & $0.56(0.46)$ & $0.47(0.42)$ & $0.53(0.43)$ & $0.86(0.29)$ & $0.81(0.36)$ & 0.15 \\
\hline Proportion of donor's human milk & $0.16(0.24)$ & $0.13(0.26)$ & $0.36(0.38)$ & $0.20(0.34)$ & $0.34(0.42)$ & $0.15(0.30)$ & $0.11(0.28)$ & $0.00(0.00)$ & 0.31 \\
\hline Proportion of formula & $0.01(0.07)$ & $0.10(0.16)$ & $0.01(0.07)$ & $0.24(0.36)$ & $0.19(0.37)$ & $0.32(0.40)$ & $0.04(0.09)$ & $0.19(0.36)$ & 0.005 \\
\hline
\end{tabular}

Data expressed as means (SD). Weight gain velocity is calculated as (current weight at stool collection-birth weight)/postnatal day of life at stool collection. The proportion of milk received is based on volume of intake. "Early" stool samples were collected $\leq 3$ weeks postnatal age, while "Late" samples were collected $>3$ weeks postnatal age. $p^{*}$ : Kruskal-Wallis comparisons between Carle Hospital (all samples) and Tampa General Hospital (all samples). Bold values indicate $p<0.01$.

\subsection{Gut Microbial Alpha Diversity}

The 16S rRNA gene sequencing of stool specimens resulted in $99.5-100 \%$ Good's coverage values [53], indicative of comprehensive microbial community sampling. The final unrarefied OTU table contained 285 OTUs from 140 individuals, including 56 singletons, 33 twin sets, and 6 triplet sets. Alpha diversity, calculated from the OTU table rarefied to 1232 OTUs per sample, did not significantly differ between NICUs, sex, or delivery method. Across all samples, both alpha diversity metrics positively correlated with weeks on BMF, corrected gestational age, current weight, and weight gain velocity, but negatively correlated with percentage of donor human milk (Supplementary Materials Figure S1). Linear mixed effects modelling revealed weeks on BMF to be a significant predictor of alpha (Shannon's and/or Faith's phylogenetic diversity) in the Carle multiplets, Carle singletons, TGH multiplets, and TGH singletons subgroups (Figure 1). Weight gain velocity was a significant predictor of Faith's phylogenetic diversity in these subgroups, except for TGH singletons, while current weight was a significant predictor of Shannon's diversity in the Carle multiplets and singletons subgroups (Figure 1). Days on antibiotics also predicted Shannon's diversity in TGH singletons, although the $p$-value was slightly above the significance threshold ( $p=0.052$; Figure 1$)$. After residualizing against clinical variables, multiplets shared significant pairwise correlations of Faith's phylogenetic diversity at early, but not late timepoints (Figure 2A). On the other hand, significant pairwise correlations of Shannon's diversity were detected at both time points from the TGH, but not Carle NICU (Figure 2B). 


\subsection{Gut Microbial Composition and Beta Diversity}

Stool samples from both Carle and TGH NICUs were dominated by the phyla Firmicutes (average 52\% relative abundance) and Proteobacteria (average $41 \%$ relative abundance) at both time points (Supplementary Materials Table S2). The 20 most abundant genera across all samples included Klebsiella, Escherichia /Shigella, Dickeya, Haemophilus, Pantoea, Proteus and unclassified taxa belonging to the class Gammaproteobacteria (Figure 3A). Abundant Firmicutes genera included Staphylococcus, Enterococcus, and Lactobacillus (class Bacilli), Clostridium sensu stricto, Lachnoclostridium, Clostridioides, and Finegoldia (class Clostridia), and Veillonella (class Negativicutes; Figure 3A). Bacteroides (phylum Bacteroidetes and class Bacteroidia) and Actinomyces (phylum Actinobacteria and class Actinobacteria) were the ninth and 20th most abundant genera across samples, respectively (Figure $3 \mathrm{~A}$ ). Twenty-three OTUs were shared across all cohorts and time points, constituting the core microbiota of preterm infants in this study (Figure 3B). These were classified to the class Actinobacteria (Varibaculum, Corynebacterium, and Cutibacterium), Coriobacteriia (Eggerthella), Bacilli (Gemella, Staphylococcus, and Enterococcus), Clostridia (Anaerococcus and Clostridium sensu stricto), Negativicutes (Negativicoccus and Veillonella), and Gammaproteobacteria (Escherichia-Shigella and other unclassified taxa).

A Significance of clinical variables in predicting Faith's phylogenetic diversity

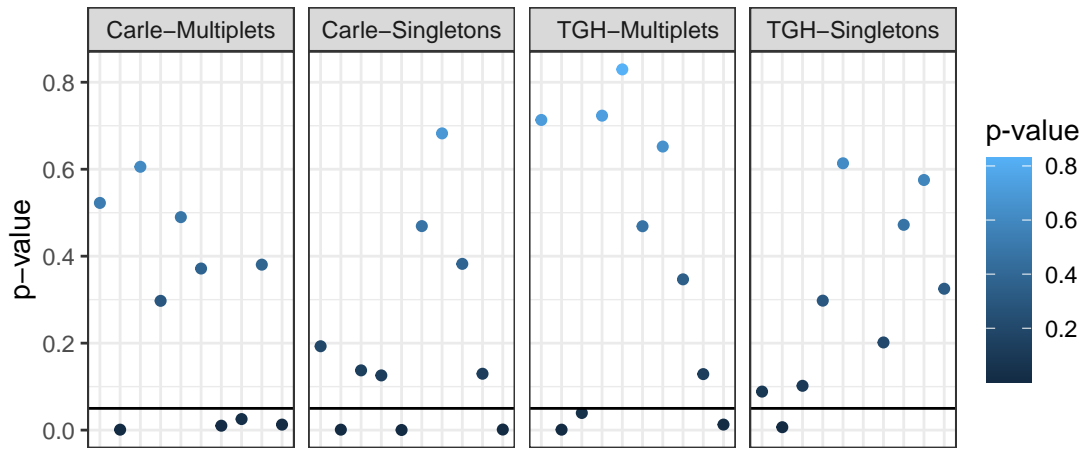

B Significance of clinical variables in predicting Shannon's diversity

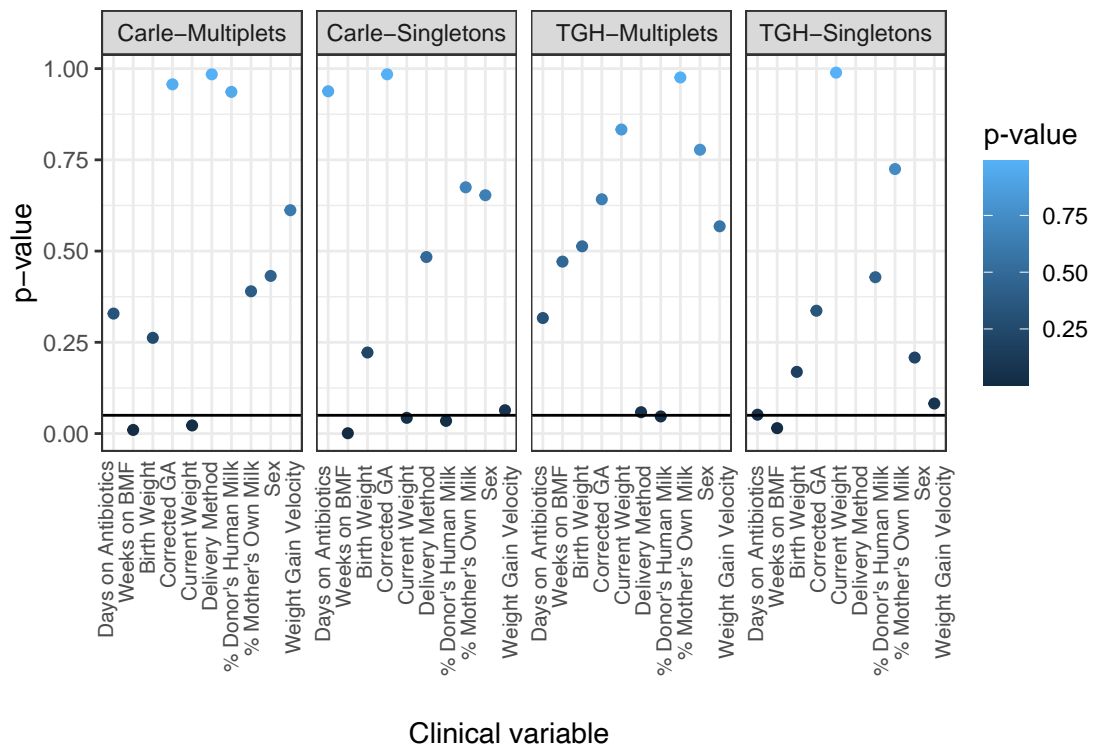

Figure 1. Significance of clinical variables in predicting (A) Faith's phylogenetic diversity and (B) Shannon's diversity, based on ANOVA testing on fitted linear mixed effects models. A horizontal line was plotted at $p=0.05$ for each subplot. Abbreviations: TGH, Tampa General Hospital; BMF, bovine milk fortifier; GA, gestational age. 


\section{Alpha diversity correlations between siblings}
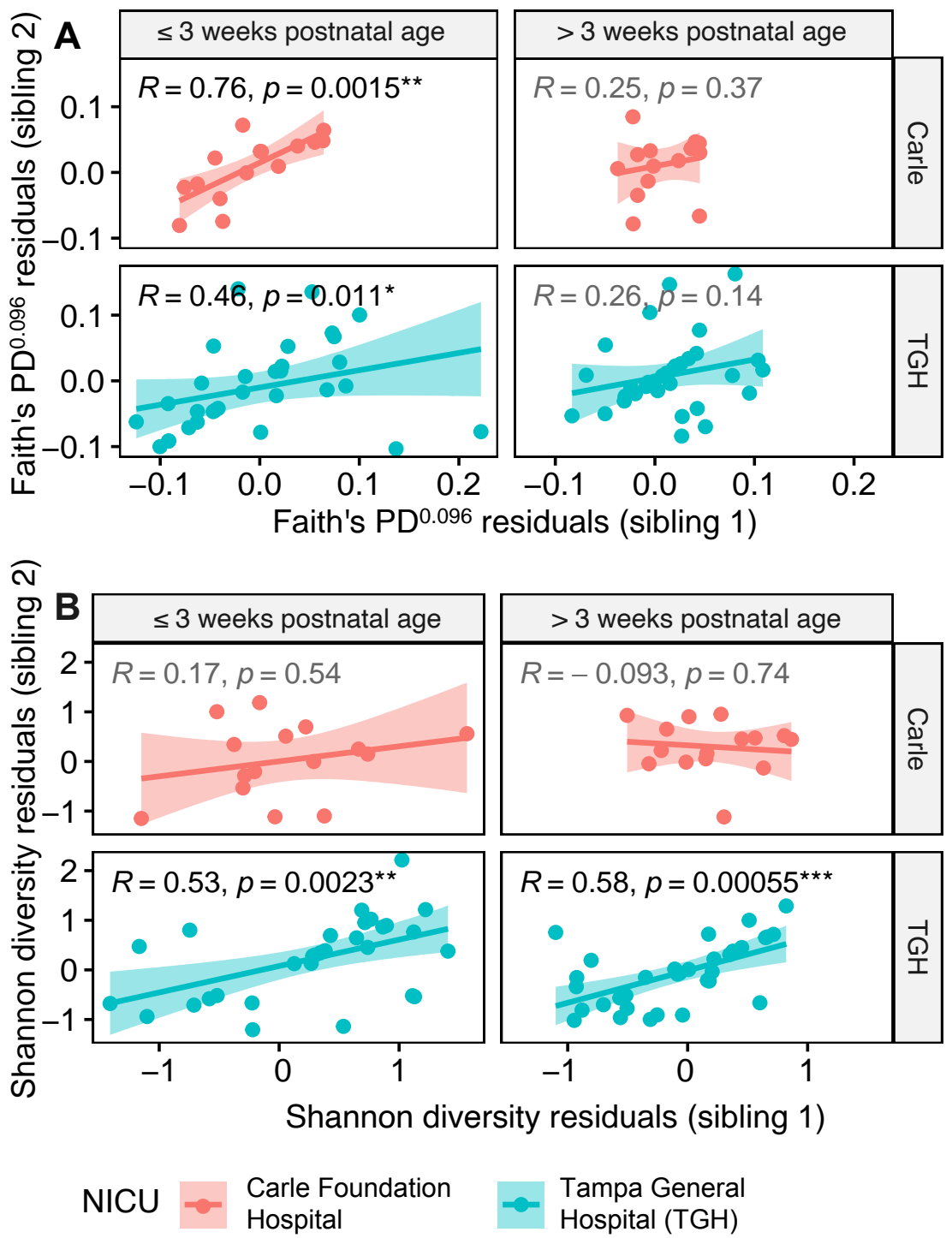

Figure 2. Pairwise correlations of residuals of (A) transformed Faith's phylogenetic diversity and (B) Shannon's diversity between siblings from both Neonatal Intensive Care Units (NICUs), after regressing out clinical variables. Statistically significant comparisons are indicated with * $(p<0.05)$, ** $(p<0.01)$, or ${ }^{* * *}(p<0.001)$.

Sixty-two OTUs were unique to the Carle NICU, while 131 were unique to the TGH NICU (Figure 3B). LDA Effect Size (LEfSe) using NICU (Carle vs. TGH) as class and sample type (multiplet vs. singleton) as subclass showed that Staphylococcus and its associated family, order, class, and phyla (Staphylococcaceae/Bacillales/Bacilli/Firmicutes) were enriched in TGH samples on/before three weeks postnatal age (Figure 3C). After three weeks postnatal age, Enterococcus, Lactobacillaceae and their associated order, class, and phyla (Bacillales/Bacilli/Firmicutes) were enriched in TGH relative to Carle samples. Gut microbial abundances were significantly influenced by weeks on BMF, current weight, and weight gain velocity in Carle singletons, TGH multiplets, and TGH singletons (Figure 4). Corrected gestational age was a significant explanatory variable of observed OTU abundances in Carle singletons and TGH multiplets (Figure 4). In Carle multiplet samples, birth weight was the only significant explanatory variable of microbial abundances (Figure 4A). 
A 20 most abundant genera across preterm infant stool samples \begin{tabular}{ll|l|l|l|l|l|l|l} 
Proteobacteria; Klebsiella - 30.3 & 33.4 & 14 & 21.1 & 18.9 & 41.6 & 0.9 & 11
\end{tabular} \begin{tabular}{|l|l|l|l|l|l|l|l|} 
Firmicutes; Staphylococcus - 25.9 & 1.1 & 30.9 & 2.4 & 39.2 & 0.6 & 29 & 0.7 \\
\hline
\end{tabular} $\begin{array}{llllllllll}\text { Proteobacteria; Escherichia-Shigella - } 6.4 & 14.9 & 15.1 & 23.6 & 5.7 & 22.5 & 11.5 & 15.2\end{array}$ \begin{tabular}{l|l|l|l|l|l|l|l|} 
Proteobacteria; Unassigned - 0.5 & 0.4 & 0 & 0.2 & 16.9 & 11 & 32 & 50.4 \\
\hline
\end{tabular} \begin{tabular}{ll|l|l|l|l|l|l|l|} 
Firmicutes; Enterococcus - 17 & 8.5 & 14.1 & 5.5 & 9.2 & 6.7 & 4.9 & 5.9
\end{tabular} \begin{tabular}{|l|l|l|l|l|l|l|l|l|}
\hline Firmicutes; Clostridium sensu stricto - 4.8 & 16.6 & 8.8 & 19.9 & 0.5 & 4.1 & 5.5 & 4.7 \\
\hline
\end{tabular} \begin{tabular}{|l|l|l|l|l|l|l|l|l|l|}
\hline Actinobacteria; Bifidobacterium - 1.9 & 13.6 & 4.2 & 7.8 & 1.1 & 3.8 & 4.5 & 2.2 \\
\hline
\end{tabular} \begin{tabular}{|l|l|l|l|l|l|l|l|l|}
\hline Firmicutes; Veillonella - 5.1 & 3.1 & 1.2 & 1.7 & 3 & 5.4 & 3.5 & 2.6 \\
\hline
\end{tabular} \begin{tabular}{|l|l|l|l|l|l|l|l|}
\hline Bacteroidetes; Bacteroides - 4.4 & 5 & 2.5 & 4.4 & 0 & 0 & 0.4 & 0 \\
\hline
\end{tabular} \begin{tabular}{|l|l|l|l|l|l|l|l|l|}
\hline Firmicutes; Streptococcus - 0.3 & 0.3 & 5 & 0.9 & 1.6 & 1 & 0.8 & 1.9 \\
\hline
\end{tabular} \begin{tabular}{|l|l|l|l|l|l|l|l|l|}
\hline Proteobacteria; Dickeya - & 0 & 0.3 & 0.1 & 4.9 & 0.3 & 0.1 & 4 & 0.3 \\
\hline
\end{tabular} \begin{tabular}{|l|l|l|l|l|l|l|l|l|}
\hline Firmicutes; Lachnoclostridium - 0 & 0 & 2.8 & 1.9 & 0 & 0 & 0.1 & 0 \\
\hline
\end{tabular} \begin{tabular}{|l|l|l|l|l|l|l|l|l|}
\hline Proteobacteria; Haemophilus - 0.4 & 0.3 & 0.4 & 1.8 & 0.5 & 0 & 0.9 & 0 \\
\hline
\end{tabular} \begin{tabular}{|l|l|l|l|l|l|l|l|l|}
\hline Firmicutes; Clostridioides - & 0 & 0.1 & 0.1 & 2.8 & 0 & 0.8 & 0 & 0.1 \\
\hline
\end{tabular} \begin{tabular}{|l|l|l|l|l|l|l|l|l|}
\hline Firmicutes; Finegoldia - & 1 & 0.7 & 0.1 & 0.2 & 0 & 0.3 & 0.1 & 0.2 \\
\hline
\end{tabular} \begin{tabular}{|l|l|l|l|l|l|l|l|l|}
\hline Proteobacteria; Pantoea - 0 & 0 & 0 & 0 & 1.1 & 0 & 0 & 0 \\
\hline
\end{tabular} \begin{tabular}{|l|l|l|l|l|l|l|l|}
\hline Proteobacteria; Escherichia - 0.3 & 0.5 & 0.1 & 0.1 & 0.1 & 0.2 & 0 & 0 \\
\hline
\end{tabular}

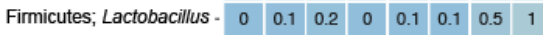
\begin{tabular}{|l|l|l|l|l|l|l|l|l|}
\hline Proteobacteria; Proteus - 0.1 & 0.1 & 0 & 0 & 0.1 & 0.4 & 0 & 0.6 \\
\hline
\end{tabular} \begin{tabular}{|l|l|l|l|l|l|l|l|}
\hline Actinobacteria; Actinomyces - 0.3 & 0 & 0 & 0 & 0 & 0.2 & 0 & 0.9 \\
\hline
\end{tabular} \begin{tabular}{l|l|l|l|l|l|l|l|l|} 
Remaining taxa (44) - 1.2 & 0.8 & 0.5 & 0.8 & 1.7 & 1.2 & 1.3 & 2.3 \\
\hline
\end{tabular} Timepoint:
Sample type:
NICU:
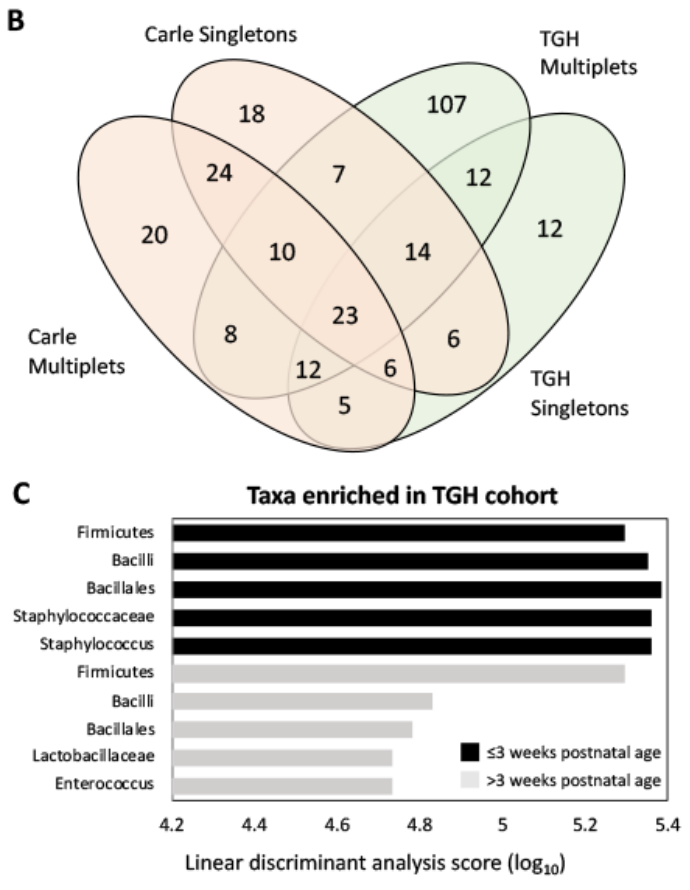

Figure 3. (A) Heatmap showing the 20 most abundant genera, and their corresponding phyla, across all samples. Cell values represent mean read abundances across samples; (B) Venn diagram showing shared operational taxonomic units (OTUs) among sample groups; and (C) histogram of the linear discriminant analysis score calculated for enriched taxa in TGH compared to Carle samples, before and after three weeks postnatal age. The score for each taxon indicates the extent of consistent relative abundance difference between Carle and TGH samples.
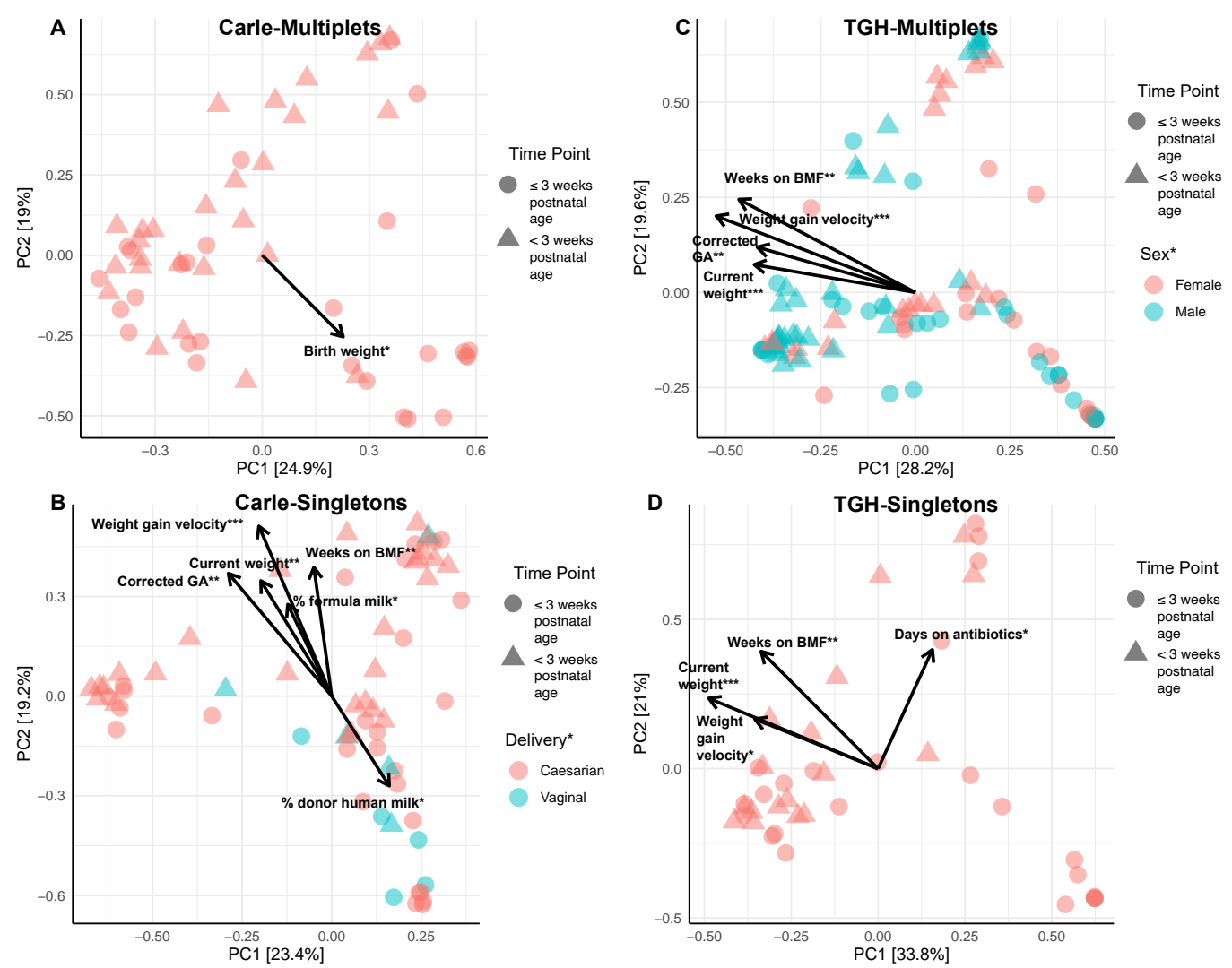

Figure 4. Principal components analysis (PCA) biplot showing the effects of clinical variables on sample clustering in (A) Carle multiplets; (B) Carle singletons; (C) Tampa General Hospital (TGH) multiplets; and (D) TGH singletons. Abbreviations: BMF, bovine milk fortifier; GA, gestational age. Statistically significant variables are indicated with ${ }^{*}(p<0.05)$, ${ }^{* *}(p<0.01)$, or $* * *(p<0.001)$. 


\subsection{Gut Microbiota Similarities between Siblings}

Compared to singletons and genetically unrelated multiplets, beta diversity Aitchison distances [51] were significantly lower between related multiplets, except for Carle samples $\leq 3$ weeks postnatal age (Figure 5A). After residualizing against clinical covariates, 30 OTUs were commonly correlated between siblings from both Carle and TGH NICUs before and after 3 weeks postnatal age (Figure 5B). These were predominantly $(40 \% ; n=15)$ Gammaproteobacteria, including OTUs classified to the families Pasteurellaceae and Enterobacteriaceae, and genera Citrobacter, Escherichia/Shigella and Klebsiella (Table 3). Other significantly correlated OTUs were classified to the genera Bifidobacterium $(n=5)$, Staphylococcus $(n=2)$, Streptococcus $(n=1)$, Bacteroides $(n=1)$, Anaerococcus $(n=1)$, Clostridium sensu stricto $(n=3)$, (Clostridium) innocuum group $(n=1)$, and Veillonella $(n=1$; Table 3$)$. In the Carle cohort, one Corynebacterium-like OTU was significantly correlated between siblings $\leq 3$ weeks postnatal age, while another Anaerococcus-like OTU was significantly correlated between siblings $>3$ weeks postnatal age (Figure 5B). Meanwhile, in the TGH cohort, 14 OTUs classified to the bacterial taxa Finegoldia, Clostridioides, Intestinibacter, Clostridium sensu stricto, Atopobium, Corynebacterium, Enterobacteriaceae, Klebsiella $(n=3)$, Dermabacter, and Cutibacterium $(n=2)$ were significantly correlated between siblings $\leq 3$ weeks postnatal age (Figure 5B). Only three OTUs classified to the genera Bifidobacterium, Streptococcus, and Staphylococcus were significantly correlated between siblings $>3$ weeks postnatal age from the TGH cohort (Figure 5B).
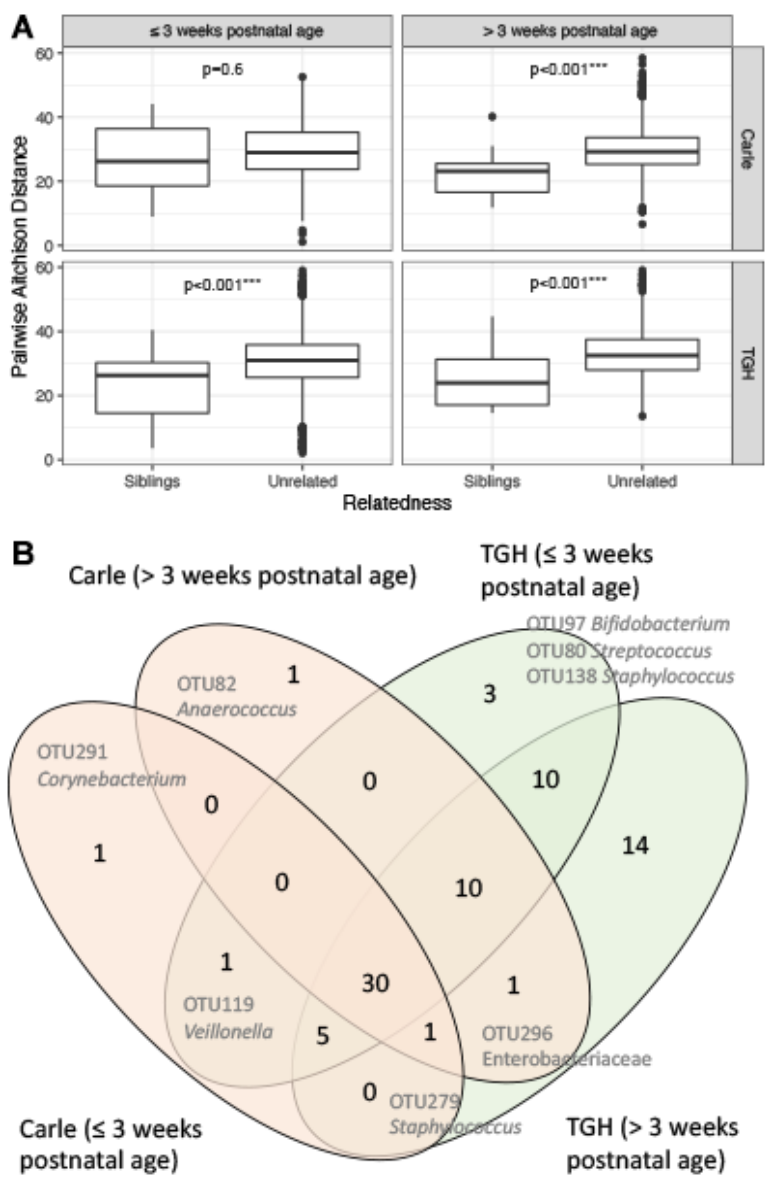

Figure 5. (A) Pairwise Aitchison distances between siblings and unrelated individuals and (B) Venn diagram showing the numbers of significantly correlated OTUs between siblings after residualizing against clinical variables. Statistically significant comparisons in $(\mathbf{A})$ are indicated with $* * *(p<0.001)$. Identifiers and taxonomic names of the 30 commonly correlated OTUs between siblings in (B) are listed in Table 2. Abbreviation: TGH, Tampa General Hospital. 
Table 3. Taxonomic classification of OTUs observed to be commonly correlated between siblings.

\begin{tabular}{|c|c|c|}
\hline OTU & Class & Family/Genus \\
\hline OTU190 & Actinobacteria & Bifidobacterium \\
\hline OTU221 & Actinobacteria & Bifidobacterium \\
\hline OTU210 & Actinobacteria & Bifidobacterium \\
\hline OTU147 & Actinobacteria & Bifidobacterium \\
\hline OTU52 & Actinobacteria & Bifidobacterium \\
\hline OTU233 & Bacilli & Staphylococcus \\
\hline OTU117 & Bacilli & Staphylococcus \\
\hline OTU176 & Bacilli & Streptococcus \\
\hline OTU6 & Bacteroidia & Bacteroides \\
\hline OTU200 & Clostridia & Anaerococcus \\
\hline OTU178 & Clostridia & Clostridium sensu stricto \\
\hline OTU28 & Clostridia & Clostridium sensu stricto \\
\hline OTU187 & Clostridia & Clostridium sensu stricto \\
\hline OTU45 & Erysipelotrichia & (Clostridium) innocuum group \\
\hline OTU283 & Gammaproteobacteria & Pasteurellaceae \\
\hline OTU284 & Gammaproteobacteria & Enterobacteriaceae \\
\hline OTU195 & Gammaproteobacteria & Enterobacteriaceae \\
\hline OTU141 & Gammaproteobacteria & Enterobacteriaceae \\
\hline OTU162 & Gammaproteobacteria & Enterobacteriaceae \\
\hline OTU222 & Gammaproteobacteria & Enterobacteriaceae \\
\hline OTU173 & Gammaproteobacteria & Enterobacteriaceae \\
\hline OTU58 & Gammaproteobacteria & Citrobacter \\
\hline OTU246 & Gammaproteobacteria & Citrobacter \\
\hline OTU272 & Gammaproteobacteria & Escherichia \\
\hline OTU234 & Gammaproteobacteria & Escherichia \\
\hline OTU105 & Gammaproteobacteria & Escherichia-Shigella \\
\hline OTU148 & Gammaproteobacteria & Escherichia-Shigella \\
\hline OTU24 & Gammaproteobacteria & Escherichia-Shigella \\
\hline OTU10 & Gammaproteobacteria & Klebsiella \\
\hline OTU133 & Negativicutes & Veillonella \\
\hline
\end{tabular}

\section{Discussion}

The preterm infant gut microbiota is dysbiotic [4-11] and perturbed by therapeutic, environmental, and endogenous factors [6,16]. Although clinical and environmental influences on preterm infant gut microbiota are relatively well-studied [6,16], the effects of genetic similarities on gut microbial diversity remain poorly understood. It is currently known that preterm siblings share higher microbiota similarities (lower beta diversity differences) compared to genetically unrelated individuals [12]. However, it is not known whether siblings also share similar alpha diversity (microbial richness and evenness), or whether observed similarities are confounded with clinical variables, such as similar diet, antibiotics exposure, or NICU exposure. In this study, we focused on examining the effects of both clinical variables and genetic relatedness on gut microbial diversity and composition in genetically related and unrelated preterm infants. To address our research objective, we analyzed the clinical data and microbial diversity of stool samples collected before and after 3 weeks postnatal age from the TGH and Carle NICUs.

Weeks on BMF and weight gain velocity were significant predictors of alpha diversity in most samples, after controlling for random effects using linear mixed effects modelling. Consistent with previous studies [4-11], preterm infant gut microbiota in our study were rich in Gammaproteobacteria OTUs, including those classified as Klebsiella, Escherichia/Shigella, Dickeya, Haemophilus, Pantoa, and Proteus. The OTU abundances (beta diversity) in most samples were similarly influenced by weeks on BMF, current weight, and weight gain velocity. Our study is one of few to report the effects of BMF on preterm infant gut microbiota, which remain poorly understood [54]. Nevertheless, several studies have cautioned that BMF may increase the risks of feeding tolerance and NEC in preterm infants $[55,56]$. Thus, connections between BMF feeding, gut microbial diversity, and clini- 
cal outcomes in preterm infants require further study. In comparison, the observed effects of weight gain on preterm infant gut microbial diversity were consistent with our previous longitudinal study focusing on a larger TGH cohort [22]. Birth weight also influenced OTU abundances in the Carle multiplet samples. However, because this effect was not consistently observed across all subgroups, the influence of birth weight may be due to random or sampling effects.

Contrary to other studies [22,23], our study observed minimal effects of mother's own milk on the preterm infant gut microbiome. Previous studies, including ours, reported positive correlations of mother's own milk with microbial alpha diversity [22,23]. Similarly, we observed significant negative correlations between alpha diversity and percentage of donor human milk, which in turn was negatively correlated with percentage of mother's own milk. However, the effects of donor human milk were non-significant after modelling for random effects. Nevertheless, we identified inter-NICU differences that could be due to different milk and milk fortifier feeding practices. Compared to Carle NICU samples, TGH NICU samples $\leq 3$ weeks postnatal age were enriched in potentially pathogenic Staphylococcus, known to contribute to neonatal sepsis [57]. Interestingly, TGH cohort had $20 \%$ rate of culture-positive sepsis versus none in the Carle cohort. After 3 weeks postnatal age, potentially pathogenic Enterococcus [58] and Lactobacillaceae were enriched in the TGH compared to Carle NICU. Overall, the TGH NICU showed greater abundance of OTUs from the order Bacillales, class Bacilli, and phylum Firmicutes across both time points. These inter-NICU differences may be related to different feeding practices, where infants born $<28$ weeks from the Carle NICU, received exclusive human milk diet, while those from the TGH NICU were exposed to bovine products from BMF. Lower abundances of Veillonella belonging to the phylum Firmicutes were similarly observed in the gut microbiota of preterm infants after 2 to 4 weeks of enteral feeding with human milk and HMF, while higher abundances of another Firmicutes genus, Terrisporobacter, were observed in those fed exclusively with formula [21]. NICU-related differences can also be confounded by different infection control practices [59] or different library preparation and sequencing strategies [60]. Future hypothesis-driven studies will be useful in examining the specific effects of HMF on preterm infant gut microbiota composition and diversity. Unlike our previous findings [22] and other studies [12,20], we did not observe significant effects of antibiotic use on preterm infant gut microbial diversity. Delivery mode [11,19] also did not significantly influence gut microbial diversity, presumably because $>80 \%$ infants in our study were delivered through cesarean section.

Besides clinical and environmental effects, we also evaluated the effects of genetic relatedness on preterm infant gut microbial diversity. Because genetic testing was not performed on infants enrolled in this study, the effects of zygosity on gut microbial diversity, which have been reported in healthy adult $[30,61,62]$ and infant [27,31] populations, were not assessed in this study. As hypothesized, we observed significantly correlated alpha diversity between siblings, especially Faith's phylogenetic diversity and in the TGH cohort, after residualizing against clinical covariates.

Although we report likely genetic influences on the preterm gut microbiota, we note that genetic effects can be confounded by shared environmental influences, such as maternal [19] or NICU inoculation [7,24]. Higher shared maternal and/or environmental influences possibly explain the higher consistency in alpha diversity correlations between siblings $\leq 3$ weeks compared to $>3$ weeks postnatal age. Shannon's diversity values were significantly correlated between siblings in TGH, but not the Carle NICU at both timepoints, which may reflect inter-NICU differences in spatial layout, or in other feeding, clinical, or environmental practices. Specifically, the open bay unit configuration in the Carle NICU likely results in increased human traffic and thus increased diversity of transmissible bacteria [63], compared to the private rooms in the TGH NICU. Consistent with previous analyses of preterm twin infant microbiota [12,32], gut microbial beta diversity profiles in siblings were more similar in siblings compared to unrelated individuals. We also identified 30 candidate "heritable" OTUs belonging to bacterial classes Actinobacteria, 
Bacilli, Bacteroidia, Clostridia, Erysipelotrichia, and Negativicutes that were commonly correlated between siblings across time points and NICUs. In the future, longitudinal studies on preterm infant multiplets will be instrumental in tracking bacterial diversity metrics and taxa that remain similar between siblings over time [64], and in linking gut microbial diversity variations to differing health outcomes. Metagenomics approaches, which offer improved taxonomic resolution [65], will also be useful in identifying specific bacterial species and strains shared between prematurely born siblings. Additionally, multiomics studies can further identify bacterial pathways and host immune-related functions that shape similarities and differences in preterm gut infant microbiota.

Despite limitations pertaining to heterogeneity, multicenter collaborations have the potential to address important clinical research questions with improved replication and results validation [66]. Our two-center study represents an effort to deepen the investigation into potential clinical and genetic determinants of preterm infant gut microbiota, with the goal of improving clinical care and the health of preterm neonates. Our findings highlight the need for future integrated and hypothesis-driven studies to disentangle the complex interactions between the preterm infant gut microbiota, maternal and NICU environment, diet, and genetics.

Supplementary Materials: The following are available online at https:/ /www.mdpi.com/2076-260 7/9/2/278/s1, Figure S1: Frequency distribution and correlation matrix of transformed clinical and alpha diversity variables. The principal diagonal shows the univariate histogram for each variable. The top half of the matrix shows the Spearman correlation values between each pair of variables, and statistically significant correlations are indicated with ${ }^{*}(p<0.05),{ }^{* *}(p<0.01)$, or ${ }^{* * *}(p<0.001)$, Figure S2: (A) Histogram of OTU total frequency in the count table prior to frequency-based filtering and (B) rarefaction curves of stool samples analyzed in this study, Table S1: List of primers used in this study, Table S2: DADA2 denoising stats and phylum relative abundances (\%) of stool samples analyzed in this study.

Author Contributions: Conceptualization, M.W.G., S.M.D. and T.H.; resources, C.W.; methodology, S.J.L.; software, S.J.L.; formal analysis, S.J.L., S.V.O.D. and M.A.-L.; data curation, M.A.-L., V.B., S.V.O.D. and S.J.L.; writing—original draft preparation, S.J.L.; writing—review and editing, M.A.-L., M.W.G., S.M.D. and T.H.; visualization, S.J.L. and S.V.O.D.; supervision, S.M.D. and T.H.; funding acquisition, M.W.G., T.H., and S.M.D. All authors have read and agreed to the published version of the manuscript.

Funding: This research was funded by NIH/NINR R21NRO13094 and RO1 NR015446 (MWG); NIH/NHLBI K23HL150300 (TH); and Carle-Illinois Collaborative Research Seed Proposal Program (TH, SMD). MAL was supported by a fellowship from the National Council of Science and Technology (CONACyT) from Mexico. SVOD received support from the Brazilian Federal Agency for Support and Evaluation of Graduate Education (CAPES) for graduate education.

Institutional Review Board Statement: The study was conducted according to the guidelines of the Declaration of Helsinki, and approved by the Institutional Review Board (or Ethics Committee) of University of South Florida (Pro00007168; date of approval 20 March 2012 for multiplets and Pro00019955; date of approval 2 December 2014 for singletons) and Carle Hospital (protocol code 15031; date of approval 18 December 2015).

Informed Consent Statement: Informed consent was obtained from the parents of all subjects involved in the study.

Data Availability Statement: The data (sequenced reads) presented in this study are publicly available on National Center for Biotechnology Information's (NCBI) Sequence Read Archive (SRA) under the BioProject accessions PRJNA449987 and PRJNA693089.

Acknowledgments: We thank research nurses Alissa MacDonald (Carle) and Judy Zaritt and Marcia Kneusel (TGH), for their valuable contributions with recruitment, and data and stool collections; Vitaly Soloveychik, Carle neonatologist, for assisting with the study; Tampa General Hospital and Carle NICU nurses for stool collection; laboratory technician, Bradley Kane, for his assistance with sample processing; and the Argonne National Laboratory for 16S rRNA library preparation. 
Conflicts of Interest: The authors declare no conflict of interest. The funders had no role in the design of the study; in the collection, analyses, or interpretation of data; in the writing of the manuscript, or in the decision to publish the results.

\section{References}

1. Hooper, L.V.; Wong, M.H.; Thelin, A.; Hansson, L.; Falk, P.G.; Gordon, J.I. Molecular analysis of commensal host-microbial relationships in the intestine. Science 2001, 291, 881-884. [CrossRef]

2. Rautava, S.; Luoto, R.; Salminen, S.; Isolauri, E. Microbial contact during pregnancy, intestinal colonization and human disease. Nat. Rev. Gastroenterol. Hepatol. 2012, 9, 565-576. [CrossRef]

3. Arboleya, S.; Martinez-Camblor, P.; Solis, G.; Suarez, M.; Fernandez, N.; de Los Reyes-Gavilan, C.G.; Gueimonde, M. Intestinal microbiota and weight-gain in preterm neonates. Front. Microbiol. 2017, 8, 183. [CrossRef]

4. Younge, N.E.; Newgard, C.B.; Cotten, C.M.; Goldberg, R.N.; Muehlbauer, M.J.; Bain, J.R.; Stevens, R.D.; O'Connell, T.M.; Rawls, J.F.; Seed, P.C.; et al. Disrupted maturation of the microbiota and metabolome among extremely preterm infants with postnatal growth failure. Sci. Rep. 2019, 9, 8167. [CrossRef]

5. Matamoros, S.; Gras-Leguen, C.; Le Vacon, F.; Potel, G.; de La Cochetiere, M.F. Development of intestinal microbiota in infants and its impact on health. Trends Microbiol. 2013, 21, 167-173. [CrossRef]

6. Groer, M.W.; Luciano, A.A.; Dishaw, L.J.; Ashmeade, T.L.; Miller, E.; Gilbert, J.A. Development of the preterm infant gut microbiome: A research priority. Microbiome 2014, 2, 38. [CrossRef]

7. Schwiertz, A.; Gruhl, B.; Lobnitz, M.; Michel, P.; Radke, M.; Blaut, M. Development of the intestinal bacterial composition in hospitalized preterm infants in comparison with breast-fed, full-term infants. Pediatr. Res. 2003, 54, 393-399. [CrossRef]

8. Morowitz, M.J.; Denef, V.J.; Costello, E.K.; Thomas, B.C.; Poroyko, V.; Relman, D.A.; Banfield, J.F. Strain-resolved community genomic analysis of gut microbial colonization in a premature infant. Proc. Natl. Acad. Sci. USA 2011, 108, 1128-1133. [CrossRef]

9. Arboleya, S.; Ang, L.; Margolles, A.; Yiyuan, L.; Dongya, Z.; Liang, X.; Solis, G.; Fernandez, N.; de Los Reyes-Gavilan, C.G.; Gueimonde, M. Deep 16S rRNA metagenomics and quantitative PCR analyses of the premature infant fecal microbiota. Anaerobe 2012, 18, 378-380. [CrossRef]

10. Arboleya, S.; Binetti, A.; Salazar, N.; Fernandez, N.; Solis, G.; Hernandez-Barranco, A.; Margolles, A.; de Los Reyes-Gavilan, C.G.; Gueimonde, M. Establishment and development of intestinal microbiota in preterm neonates. FEMS Microbiol. Ecol. 2012, 79, 763-772. [CrossRef]

11. Ward, D.V.; Scholz, M.; Zolfo, M.; Taft, D.H.; Schibler, K.R.; Tett, A.; Segata, N.; Morrow, A.L. Metagenomic sequencing with strain-level resolution implicates uropathogenic E. coli in necrotizing enterocolitis and mortality in preterm infants. Cell Rep. 2016, 14, 2912-2924. [CrossRef]

12. Stewart, C.J.; Marrs, E.C.; Nelson, A.; Lanyon, C.; Perry, J.D.; Embleton, N.D.; Cummings, S.P.; Berrington, J.E. Development of the preterm gut microbiome in twins at risk of necrotising enterocolitis and sepsis. PLoS ONE 2013, 8, e73465. [CrossRef]

13. Bertelli, C.; Pillonel, T.; Torregrossa, A.; Prodhom, G.; Fischer, C.J.; Greub, G.; Giannoni, E. Bifidobacterium longum bacteremia in preterm infants receiving probiotics. Clin. Infect. Dis. 2015, 60, 924-927. [CrossRef]

14. Mancano, M.A. Bacteremia in preterm infants receiving probiotics; throbbing headache associated with Enoxaparin use; DRESS reaction following isoniazid treatment; SIADH associated with Glimepiride; mania associated with Bortezomib administration. Hosp. Pharm. 2015, 50, 441-445. [CrossRef]

15. Rao, S.C.; Athalye-Jape, G.K.; Deshpande, G.C.; Simmer, K.N.; Patole, S.K. Probiotic supplementation and late-onset sepsis in preterm infants: A meta-analysis. Pediatrics 2016, 137, e20153684. [CrossRef]

16. Staude, B.; Oehmke, F.; Lauer, T.; Behnke, J.; Gopel, W.; Schloter, M.; Schulz, H.; Krauss-Etschmann, S.; Ehrhardt, H. The microbiome and preterm birth: A change in paradigm with profound implications for pathophysiologic concepts and novel therapeutic strategies. Biomed. Res. Int. 2018, 2018, 7218187. [CrossRef]

17. Gupta, R.W.; Tran, L.; Norori, J.; Ferris, M.J.; Eren, A.M.; Taylor, C.M.; Dowd, S.E.; Penn, D. Histamine-2 receptor blockers alter the fecal microbiota in premature infants. J. Pediatr. Gastroenterol. Nutr. 2013, 56, 397-400. [CrossRef]

18. Keski-Nisula, L.; Kyynarainen, H.R.; Karkkainen, U.; Karhukorpi, J.; Heinonen, S.; Pekkanen, J. Maternal intrapartum antibiotics and decreased vertical transmission of Lactobacillus to neonates during birth. Acta Paediatr. 2013, 102, 480-485. [CrossRef]

19. Arboleya, S.; Sanchez, B.; Milani, C.; Duranti, S.; Solis, G.; Fernandez, N.; de los Reyes-Gavilan, C.G.; Ventura, M.; Margolles, A.; Gueimonde, M. Intestinal microbiota development in preterm neonates and effect of perinatal antibiotics. J. Pediatr. 2015, 166, 538-544. [CrossRef]

20. Greenwood, C.; Morrow, A.L.; Lagomarcino, A.J.; Altaye, M.; Taft, D.H.; Yu, Z.; Newburg, D.S.; Ward, D.V.; Schibler, K.R. Early empiric antibiotic use in preterm infants is associated with lower bacterial diversity and higher relative abundance of Enterobacter. J. Pediatr. 2014, 165, 23-29. [CrossRef]

21. Cai, C.; Zhang, Z.; Morales, M.; Wang, Y.; Khafipour, E.; Friel, J. Feeding practice influences gut microbiome composition in very low birth weight preterm infants and the association with oxidative stress: A prospective cohort study. Free Radic. Biol. Med. 2019, 142, 146-154. [CrossRef]

22. Yee, A.L.; Miller, E.; Dishaw, L.J.; Gordon, J.M.; Ji, M.; Dutra, S.; Ho, T.T.B.; Gilbert, J.A.; Groer, M. Longitudinal microbiome composition and stability correlate with increased weight and length of very-low-birth-weight infants. MSystems 2019, 4. [CrossRef] 
23. Zanella, A.; Silveira, R.C.; Roesch, L.F.W.; Corso, A.L.; Dobbler, P.T.; Mai, V.; Procianoy, R.S. Influence of own mother's milk and different proportions of formula on intestinal microbiota of very preterm newborns. PLoS ONE 2019, 14, e0217296. [CrossRef]

24. Brooks, B.; Firek, B.A.; Miller, C.S.; Sharon, I.; Thomas, B.C.; Baker, R.; Morowitz, M.J.; Banfield, J.F. Microbes in the neonatal intensive care unit resemble those found in the gut of premature infants. Microbiome 2014, 2, 1. [CrossRef]

25. Romero, R.; Dey, S.K.; Fisher, S.J. Preterm labor: One syndrome, many causes. Science 2014, 345, 760-765. [CrossRef]

26. Nanthakumar, N.; Meng, D.; Goldstein, A.M.; Zhu, W.; Lu, L.; Uauy, R.; Llanos, A.; Claud, E.C.; Walker, W.A. The mechanism of excessive intestinal inflammation in necrotizing enterocolitis: An immature innate immune response. PLoS ONE 2011, 6, e17776. [CrossRef]

27. Turnbaugh, P.J.; Hamady, M.; Yatsunenko, T.; Cantarel, B.L.; Duncan, A.; Ley, R.E.; Sogin, M.L.; Jones, W.J.; Roe, B.A.; Affourtit, J.P.; et al. A core gut microbiome in obese and lean twins. Nature 2009, 457, 480-484. [CrossRef]

28. Lee, S.; Sung, J.; Lee, J.; Ko, G. Comparison of the gut microbiotas of healthy adult twins living in South Korea and the United States. Appl. Environ. Microbiol. 2011, 77, 7433-7437. [CrossRef] [PubMed]

29. Tims, S.; Derom, C.; Jonkers, D.M.; Vlietinck, R.; Saris, W.H.; Kleerebezem, M.; de Vos, W.M.; Zoetendal, E.G. Microbiota conservation and BMI signatures in adult monozygotic twins. ISME J. 2013, 7, 707-717. [CrossRef]

30. Goodrich, J.K.; Waters, J.L.; Poole, A.C.; Sutter, J.L.; Koren, O.; Blekhman, R.; Beaumont, M.; Van Treuren, W.; Knight, R.; Bell, J.T.; et al. Human genetics shape the gut microbiome. Cell 2014, 159, 789-799. [CrossRef]

31. Lim, E.S.; Zhou, Y.; Zhao, G.; Bauer, I.K.; Droit, L.; Ndao, I.M.; Warner, B.B.; Tarr, P.I.; Wang, D.; Holtz, L.R. Early life dynamics of the human gut virome and bacterial microbiome in infants. Nat. Med. 2015, 21, 1228-1234. [CrossRef] [PubMed]

32. Costello, E.K.; Carlisle, E.M.; Bik, E.M.; Morowitz, M.J.; Relman, D.A. Microbiome assembly across multiple body sites in low-birthweight infants. mBio 2013, 4, e00782-13. [CrossRef] [PubMed]

33. Li, M.; Bauer, L.L.; Chen, X.; Wang, M.; Kuhlenschmidt, T.B.; Kuhlenschmidt, M.S.; Fahey, G.C., Jr.; Donovan, S.M. Microbial composition and in vitro fermentation patterns of human milk oligosaccharides and prebiotics differ between formula-fed and sow-reared piglets. J. Nutr. 2012, 142, 681-689. [CrossRef] [PubMed]

34. Bolyen, E.; Rideout, J.R.; Dillon, M.R.; Bokulich, N.A.; Abnet, C.C.; Al-Ghalith, G.A.; Alexander, H.; Alm, E.J.; Arumugam, M.; Asnicar, F; et al. Reproducible, interactive, scalable and extensible microbiome data science using QIIME 2. Nat. Biotechnol. 2019, 37, 852-857. [CrossRef]

35. Callahan, B.J.; McMurdie, P.J.; Rosen, M.J.; Han, A.W.; Johnson, A.J.; Holmes, S.P. DADA2: High-resolution sample inference from Illumina amplicon data. Nat. Methods 2016, 13, 581-583. [CrossRef]

36. Li, W.; Godzik, A. CD-HIT: A fast program for clustering and comparing large sets of protein or nucleotide sequences. Bioinformatics 2006, 22, 1658-1659. [CrossRef]

37. Quast, C.; Pruesse, E.; Yilmaz, P.; Gerken, J.; Schweer, T.; Yarza, P.; Peplies, J.; Glockner, F.O. The SILVA ribosomal RNA gene database project: Improved data processing and web-based tools. Nucleic Acids Res. 2013, 41, D590-D596. [CrossRef]

38. Knight, R.; Vrbanac, A.; Taylor, B.C.; Aksenov, A.; Callewaert, C.; Debelius, J.; Gonzalez, A.; Kosciolek, T.; McCall, L.I.; McDonald, D.; et al. Best practices for analysing microbiomes. Nat. Rev. Microbiol. 2018, 16, 410-422. [CrossRef]

39. Janssen, S.; McDonald, D.; Gonzalez, A.; Navas-Molina, J.A.; Jiang, L.; Xu, Z.Z.; Winker, K.; Kado, D.M.; Orwoll, E.; Manary, M.; et al. Phylogenetic placement of exact amplicon sequences improves associations with clinical information. MSystems 2018, 3. [CrossRef]

40. Sayers, E.W.; Beck, J.; Brister, J.R.; Bolton, E.E.; Canese, K.; Comeau, D.C.; Funk, K.; Ketter, A.; Kim, S.; Kimchi, A.; et al. Database resources of the National Center for Biotechnology Information. Nucleic Acids Res. 2020, 48, D9-D16. [CrossRef]

41. Drancourt, M.; Bollet, C.; Carlioz, A.; Martelin, R.; Gayral, J.P.; Raoult, D. 16S ribosomal DNA sequence analysis of a large collection of environmental and clinical unidentifiable bacterial isolates. J. Clin. Microbiol. 2000, 38, 3623-3630. [CrossRef] [PubMed]

42. Shannon, C.E. A mathematical theory of communication. Bell Syst. Techn. J. 1948, 27, 379-423. [CrossRef]

43. Pielou, E.C. The measurement of diversity in different types of biological collections. J. Theor. Biol. 1966, 13, 131-144. [CrossRef]

44. Faith, D.P. Conservation evaluation and phylogenetic diversity. Biol. Conserv. 1992, 61, 1-10. [CrossRef]

45. Fox, J.; Weisberg, S. An R Companion to Applied Regression, 3rd ed.; SAGE Publications, Inc.: Thousand Oaks, CA, USA, 2018; p. 608.

46. Bates, D.; Mächler, M.; Bolker, B.; Walker, S. Fitting linear mixed-effects models using lme4. J. Stat. Softw. 2015, 67, 48. [CrossRef]

47. Akaike, H. Information theory and an extension of the maximum likelihood principle. In Selected Papers of Hirotugu Akaike; Parzen, E., Tanabe, K., Kitagawa, G., Eds.; Springer: New York, NY, USA, 1998; pp. 199-213. [CrossRef]

48. Andersen, K.S.; Kirkegaard, R.H.; Karst, S.M.; Albertsen, M. Ampvis2: An R package to analyse and visualise 16S rRNA amplicon data. bioRxiv 2018, 299537. [CrossRef]

49. Legendre, P.; Gallagher, E.D. Ecologically meaningful transformations for ordination of species data. Oecologia 2001, 129, 271-280. [CrossRef]

50. Segata, N.; Izard, J.; Waldron, L.; Gevers, D.; Miropolsky, L.; Garrett, W.S.; Huttenhower, C. Metagenomic biomarker discovery and explanation. Genome Biol. 2011, 12, R60. [CrossRef]

51. Aitchison, J.; Barceló-Vidal, C.; Martín-Fernández, J.A.; Pawlowsky-Glahn, V. Logratio analysis and compositional distance. Math. Geol. 2000, 32, 271-275. [CrossRef]

52. Benjamini, Y.; Hochberg, Y. Controlling the false discovery rate: A practical and powerful approach to multiple testing. J. R. Stat. Soc. Ser. B 1995, 57, 289-300. [CrossRef] 
53. Good, I.J. The population frequencies of species and the estimation of population parameters. Biometrika 1953, 40, 237-264. [CrossRef]

54. Granger, C.L.; Embleton, N.D.; Palmer, J.M.; Lamb, C.A.; Berrington, J.E.; Stewart, C.J. Maternal breastmilk, infant gut microbiome and the impact on preterm infant health. Acta Paediatr. 2020, 110, 2. [CrossRef] [PubMed]

55. Hammond, P.J.; Flett, M.E.; De La Hunt, M. Fulminant necrotising enterocolitis immediately following change to low birth weight formula feeds. Eur. J. Pediatr. Surg. 2008, 18, 185-187. [CrossRef] [PubMed]

56. Mimouni, F.B.; Nathan, N.; Ziegler, E.E.; Lubetzky, R.; Mandel, D. The use of multinutrient human milk fortifiers in preterm infants: A systematic review of unanswered questions. Clin. Perinatol. 2017, 44, 173-178. [CrossRef] [PubMed]

57. Dong, Y.; Speer, C.P. Late-onset neonatal sepsis: Recent developments. Arch. Dis. Child. Fetal. Neonatal. Ed. 2015, 100, F257-F263. [CrossRef]

58. Hufnagel, M.; Liese, C.; Loescher, C.; Kunze, M.; Proempeler, H.; Berner, R.; Krueger, M. Enterococcal colonization of infants in a neonatal intensive care unit: Associated predictors, risk factors and seasonal patterns. BMC Infect. Dis. 2007, 7, 107. [CrossRef]

59. Shane, A.L.; Hansen, N.I.; Stoll, B.J.; Bell, E.F.; Sanchez, P.J.; Shankaran, S.; Laptook, A.R.; Das, A.; Walsh, M.C.; Hale, E.C.; et al. Methicillin-resistant and susceptible Staphylococcus aureus bacteremia and meningitis in preterm infants. Pediatrics 2012, 129, e914-e922. [CrossRef]

60. Jones, M.B.; Highlander, S.K.; Anderson, E.L.; Li, W.; Dayrit, M.; Klitgord, N.; Fabani, M.M.; Seguritan, V.; Green, J.; Pride, D.T.; et al. Library preparation methodology can influence genomic and functional predictions in human microbiome research. Proc. Natl. Acad. Sci. USA 2015, 112, 14024-14029. [CrossRef]

61. Yatsunenko, T.; Rey, F.E.; Manary, M.J.; Trehan, I.; Dominguez-Bello, M.G.; Contreras, M.; Magris, M.; Hidalgo, G.; Baldassano, R.N.; Anokhin, A.P.; et al. Human gut microbiome viewed across age and geography. Nature 2012, 486, 222-227. [CrossRef]

62. Goodrich, J.K.; Davenport, E.R.; Beaumont, M.; Jackson, M.A.; Knight, R.; Ober, C.; Spector, T.D.; Bell, J.T.; Clark, A.G.; Ley, R.E Genetic determinants of the gut microbiome in UK Twins. Cell Host Microbe 2016, 19, 731-743. [CrossRef]

63. Hewitt, K.M.; Mannino, F.L.; Gonzalez, A.; Chase, J.H.; Caporaso, J.G.; Knight, R.; Kelley, S.T. Bacterial diversity in two Neonatal Intensive Care Units (NICUs). PLoS ONE 2013, 8, e54703. [CrossRef] [PubMed]

64. Koo, H.; Hakim, J.A.; Crossman, D.K.; Lefkowitz, E.J.; Morrow, C.D. Sharing of gut microbial strains between selected individual sets of twins cohabitating for decades. PLoS ONE 2019, 14, e0226111. [CrossRef] [PubMed]

65. Ranjan, R.; Rani, A.; Metwally, A.; McGee, H.S.; Perkins, D.L. Analysis of the microbiome: Advantages of whole genome shotgun versus 16S amplicon sequencing. Biochem. Biophys. Res. Commun. 2016, 469, 967-977. [CrossRef] [PubMed]

66. Youseff, N.; Reinhart, K.; Sakr, Y. The pros and cons of multicentre studies. Neth. J. Crit Care 2008, 12, 120-122. 